Courrier du Centre international Blaise Pascal

$28 \mid 2006$

Varia

\title{
Le Mémorial
}

Autour de la commémoration de la «nuit de feu »

\section{Dominique Descotes}

\section{OpenEdition}

Journals

Édition électronique

URL : http://journals.openedition.org/ccibp/503

DOI : $10.4000 /$ ccibp.503

ISSN : 2493-7460

Éditeur

Centre international Blaise Pascal

\section{Édition imprimée}

Date de publication : 16 octobre 2006

Pagination : 2-3

ISBN : 2-84516-305-3

ISSN : 0249-6674

\section{Référence électronique}

Dominique Descotes, « Le Mémorial », Courrier du Centre international Blaise Pascal [En ligne], 28 | 2006, mis en ligne le 02 décembre 2015, consulté le 24 septembre 2020. URL : http:// journals.openedition.org/ccibp/503; DOI : https://doi.org/10.4000/ccibp.503

Ce document a été généré automatiquement le 24 septembre 2020.

Centre international Blaise Pascal 


\title{
Le Mémorial
}

\author{
Autour de la commémoration de la «nuit de feu »
}

\section{Dominique Descotes}

1 Le trois cent cinquantième anniversaire du Mémorial a donné lieu à plusieurs manifestations, à Paris et à Clermont principalement.

2 Le Centre International Blaise Pascal a organisé à la Maison de la Recherche de Clermont-Ferrand (4 rue Ledru) une manifestation scientifique centrée sur la nuit de $\mathrm{feu}$, mais aussi, plus largement, sur le thème de la conversion. La matinée du 26 Novembre 2004 a été consacrée aux différents aspects du Mémorial. Laurent Thirouin a consacré son intervention au couple «se convertir, se divertir ", dont il a montré l'opposition, mais aussi la parenté profonde dans la pensée de Pascal. Jean Mesnard a présenté une étude qui prolongeait les analyses de son édition, sur Bible et liturgie dans le Mémorial. Hélène Michon a ensuite traité de l'écriture mystique du Mémorial, dans le prolongement des réflexions de H. Gouhier sur ce sujet inépuisable. L'après-midi a élargi les perspectives : Laurence Devillairs a présenté les grands traits qui opposent Fénelon à Pascal. Puis Emmanuèle Lesne-Jaffro nous a carrément fait passer les océans, pour examiner des cas de conversions dans le Nouveau Monde, qui révélaient le retentissement qu'elles suscitaient non pas seulement chez les candidats à la conversion, mais surtout chez les convertisseurs eux-mêmes. Enfin, pour rendre hommage à l'un des maîtres des études pascaliennes, Emmanuelle Tabet a traité de la conversion dans l'œuvre de Sainte-Beuve, des Consolations à Port-Royal. L'assistance était nombreuse, attentive et assidue ; Monseigneur Hippolyte Simon, archevêque de Clermont, nous a fait l'honneur d'y assister avec une particulière attention. Vers 18 heures 30, une réception donnée à l'Hôtel de Ville pour tous les participants du colloque a permis à Antony McKenna d'offrir à M. Dantal, adjoint au Maire chargé de la recherche, un exemplaire du Dictionnaire de Port-Royal tout récemment publié, en soulignant la part que le Centre International Blaise Pascal a prise dans sa composition, notamment dans le domaine iconographique. Jean Fau a profité de cette occasion pour rappeler à nos autorités de tutelle les buts et les réalisations récentes du CIBP.

3 Le comité d'animation réuni le 29 Mars 2004 s'était demandé quelle sorte de célébration ou de spectacle était susceptible d'attirer sur l'anniversaire du Mémorial l'attention du 
public. Il a été décidé de reprendre, en l'adaptant à la circonstance, le concert donné à la cathédrale de Clermont-Ferrand en 2002, où la déclamation des textes des Pensées était accompagnée et illustrée par la musique des grandes orgues et des chants de la chorale. Le concert a eu lieu à Saint-Genest des Carmes. La Schola Saint-Genès, la chorale diocésaine, l'ensemble vocal «Blaise Pascal », avec ses solistes, Céline Lacroix, soprano, et Philippe Gayou, ténor, dirigés par Blaise Plumettaz avec à l'orgue François Clément, ont servi avec ferveur les chants qui soulignaient les textes. La prestation de Josépha Jeunet pour le texte du Mémorial, qui était une innovation par rapport au concert de 2002, était particulièrement émouvante. M. Bernard Pourreyron sut partager avec elle la difficile tâche de faire entendre les textes des Pensées. Les pièces musicales et compositions originales de François Clément à l'orgue ont également été très appréciées par un public nombreux et conquis. Monseigneur Hippolyte Simon, a aussi marqué par sa présence l'intérêt qu'il porte à Blaise Pascal, et M. François Robert, directeur de la mission culturelle de la Ville, était là avec son épouse, représentant M. le Maire.

Quelques jours plus tôt, le mardi 23 novembre 2004, en l'église Saint-Jacques du HautPas, à Paris, s'était déroulée, toujours pour le $350^{\circ}$ anniversaire de la conversion de Pascal, une Veillée du Mémorial, organisée sous le patronage du Ministère de la Culture et de la communication et la Société des Amis de Port-Royal. L'ouverture en a été assurée par le P. Jean-Noël Bezançon, curé de Saint-Jacques du-Haut-Pas. La première partie de la soirée a été consacrée à la lecture de textes de Pascal et de récits de sa famille, dits par des artistes, et commentés par Jean Mesnard, avec projection des deux manuscrits $\mathrm{du}$ Mémorial. La deuxième partie proposait quelques témoignages relatifs au retentissement qu'avait eu le Mémorial : ont successivement pris la parole Jean Foyer, Philippe Sellier, Hélène Michon, et Gérard Ferreyrolles. Des intermèdes à l'orgue accompagnaient la parole. Une allocution du P. Jean-Robert Armogathe a achevé la veillée.

Voici quelques-uns des témoignages apportés au cours de cette soirée de méditation.

\section{INDEX}

Mots-clés : Pascal, Mémorial

\section{AUTEUR}

DOMINIQUE DESCOTES

CERHAC. Université Blaise Pascal 